PREPARED FOR THE U.S. DEPARTMENT OF ENERGY, UNDER CONTRACT DE-AC02-76CH03073

PPPL-3679

PPPL-3679

UC-70

Magnetic Propulsion of Intense Lithium Streams in a Tokamak Magnetic Field

by

Leonid E. Zakharov

March 2002

$\left.\stackrel{M}{M}\right|_{\substack{\text { PRInCETON PLASIMA } \\ \text { PHYSICS LABORATORY }}} ^{D}$

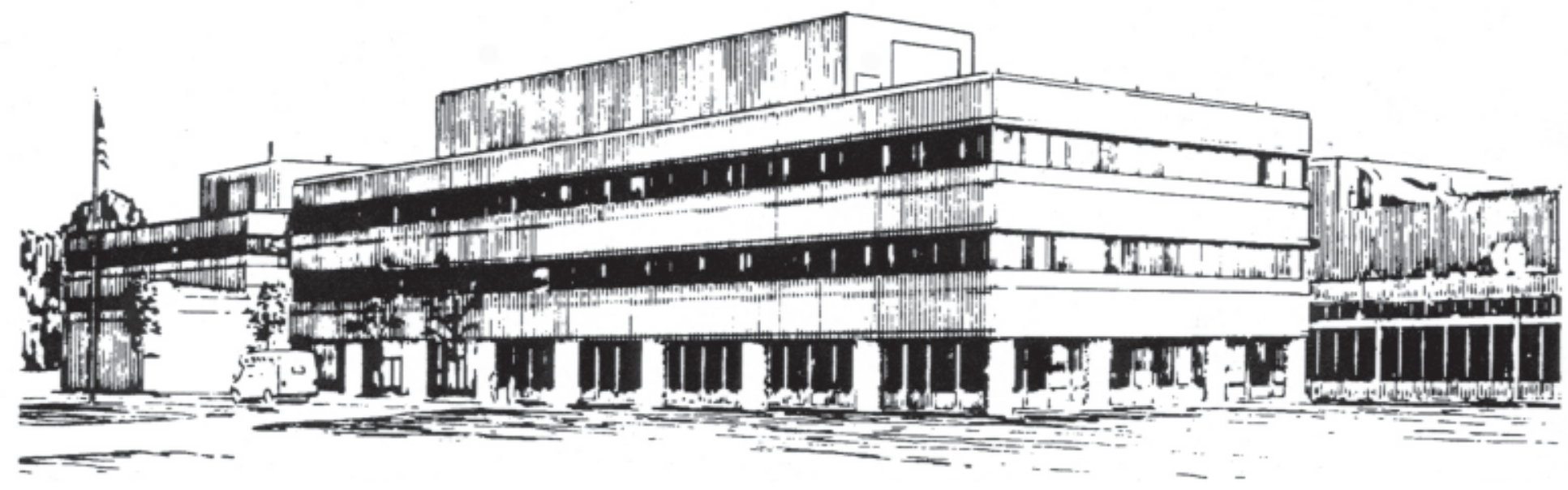

PRINCETON PLASMA PHYSICS LABORATORY PRINCETON UNIVERSITY, PRINCETON, NEW JERSEY 


\section{PPPL Reports Disclaimer}

This report was prepared as an account of work sponsored by an agency of the United States Government. Neither the United States Government nor any agency thereof, nor any of their employees, makes any warranty, express or implied, or assumes any legal liability or responsibility for the accuracy, completeness, or usefulness of any information, apparatus, product, or process disclosed, or represents that its use would not infringe privately owned rights. Reference herein to any specific commercial product, process, or service by trade name, trademark, manufacturer, or otherwise, does not necessarily constitute or imply its endorsement, recommendation, or favoring by the United States Government or any agency thereof. The views and opinions of authors expressed herein do not necessarily state or reflect those of the United States Government or any agency thereof.

\section{Availability}

This report is posted on the U.S. Department of Energy's Princeton Plasma Physics Laboratory Publications and Reports web site in Fiscal Year 2002. The home page for PPPL Reports and Publications is: http://www.pppl.gov/pub_report/

DOE and DOE Contractors can obtain copies of this report from:

U.S. Department of Energy

Office of Scientific and Technical Information

DOE Technical Information Services (DTIS)

P.O. Box 62

Oak Ridge, TN 37831

Telephone: (865) 576-8401

Fax: (865) 576-5728

Email: reports@adonis.osti.gov

This report is available to the general public from:

National Technical Information Service

U.S. Department of Commerce

5285 Port Royal Road

Springfield, VA 22161

Telephone: 1-800-553-6847 or

(703) 605-6000

Fax: (703) 321-8547

Internet: http://www.ntis.gov/ordering.htm 


\title{
Magnetic Propulsion of Intense Lithium Streams in a Tokamak Magnetic Field
}

\author{
Leonid E. Zakharov \\ Princeton Plasma Physics Laboratory, Princeton, NJ 08543-0451
}

(February 25, 2002)

\begin{abstract}
The paper gives the theory of magnetic propulsion of liquid lithium streams and their stability in tokamaks. In the approximation of a thin flowing layer the MHD equations are reduced to one integro-differential equation which takes into account the propulsion effect, viscosity and the drag force due to magnetic pumping and other interactions with the magnetic field. A criterion is obtained for stabilization of the "sausage" instability of the streams by centrifugal force.
\end{abstract}

52.55.Fa, 52.30.Jb, 52.35.Py, 52.65.Kj

Typeset using REVTEX 
For fusion, liquid lithium has unique physical properties (low melting temperature of $T_{\text {melt }} \simeq 180^{\circ} \mathrm{C}$, low density $\rho \simeq 0.53 \mathrm{~g} / \mathrm{cm}^{3}$, low viscosity $\nu \simeq 0.0004 \mathrm{~Pa} \cdot \mathrm{sec}$ in combination with high thermal capacity $c_{p} \simeq 4200 \mathrm{~J} /(\mathrm{kg} \cdot \mathrm{K})$ and thermal conductivity $\kappa \simeq 47 \mathrm{~W} /(\mathrm{m} \cdot \mathrm{K})$ [1]) which would make it potentially the best coolant for blankets of thermonuclear devices. Nevertheless, because of the rather high electric conductivity, $\left.\sigma \simeq 3.3 \cdot 10^{6}(\Omega \cdot m)^{-1}[2]\right)$ liquid lithium has been essentially dropped from the design of magnetic fusion reactors. In the strong magnetic field of tokamaks it is very energy consuming to drive a lithium flow if it is restrained by pipe walls or channels. Existing proposals for using so-called "liquid walls" for power extraction from the tokamak reactor plasma [3] rely on passive lithium flow which is sensitive to a variety of MHD effects. A recently discovered (December, 1998) effect of magnetic propulsion suggests another way of using lithium, i.e., with the actively driven free surface streaming along the inner walls of the vacuum chamber.

This paper outlines the theoretical basis of magnetic propulsion. Fig. 1a shows two lithium layers of about $1 \mathrm{~cm}$ thick, which are held to the surface by the $\mathbf{I}_{\text {pol }} \times \mathbf{B}_{\text {tor }}$ electromagnetic force, where $\mathbf{I}_{p o l}$ represents an externally driven electric current through the layers and $\mathbf{B}_{\text {tor }}$ is the toroidal magnetic field of the tokamak. The pressure $p$ in the fluid, created by the electromagnetic force, is inhomogeneous in both normal and longitudinal directions. In the normal direction it is analogous to the atmospheric (highly magnified) pressure of the Earth. It vanishes at the free surface of the liquid and reaches maximum (about one or several atmospheres) at the surface of the wall. Its inhomogeneity along the wall surface propels the fluid (as a wind) from the high field side of the chamber, where the $\mathbf{I}_{\text {pol }} \times \mathbf{B}_{\text {tor }}$ force is largest, to the low field side.

Neglecting viscosity and possible electromagnetic drag forces, the fluid velocity $\mathbf{V}$ in each flowing layer obeys the Bernoulli law

$$
p+\rho g z+\rho \frac{\mathbf{V}^{2}}{2}=\text { const }, \quad p=\left|\mathbf{I}_{\text {pol }} \times \mathbf{B}_{\text {tor }}\right|,
$$

where $z$ is the vertical coordinate and $g=9.8 \mathrm{~m} / \mathrm{sec}^{2}$ is the acceleration of gravity. Note, that the tokamak magnetic field $\mathbf{B}_{t o r}=5 \mathrm{~T}$, reasonable for reactors, corresponds to a 
magnetic pressure of $100 \mathrm{~atm}$. Using only a small fraction of it (e.g., 1-2 \%) it is possible to create a pressure drop along the flow larger than $1 \mathrm{~atm}$, which corresponds to $\simeq 20 \mathrm{~m} / \mathrm{sec}$ of the liquid lithium velocity. Such a velocity is sufficient to provide reactor relevant power extraction from the plasma of the order of $3.5 \mathrm{MW} / \mathrm{m}^{2}$. For comparison, in the International Thermonuclear Experimental Reactor project this number is about 20 times smaller [4].

This paper describes the propulsion itself. Two closely related problems of how to supply the liquid to the inlet (e.g., in the form of fast thin jets and their conversion into the flow) or to remove it from the chamber (e.g., by converting the flow into a jet shower) are not considered here.

The dynamics of the liquid metal is determined by a standard set of MHD equations

$$
\begin{aligned}
& \rho \frac{\partial \mathbf{V}}{\partial t}+\rho(\mathbf{V} \cdot \nabla) \mathbf{V}=-\nabla p-\rho g \nabla z+(\mathbf{j} \times \mathbf{B})+\nu \Delta \mathbf{V} \\
& \frac{\partial \mathbf{A}}{\partial t}-\nabla \varphi_{E}+(\mathbf{V} \times \mathbf{B})=\frac{\mathbf{j}}{\sigma}
\end{aligned}
$$

where $\mathbf{A}$ is the vector potential of the magnetic field, $\varphi_{E}$ is the electric potential, $\mathbf{j}$ is the current density in the metal and $\nu$ is the viscosity.

The toroidal geometry of tokamaks justifies the consideration of axisymmetric flow with no side walls limiting the streams. Note, that even with a few separating walls present, the magnetic propulsion is not sensitive to the resulting Hartmann drag force [5].

We use the orthogonal curvilinear coordinates $n, s, \phi$

$$
r=r_{w}-z_{w}^{\prime} n, \quad z=z_{w}+r_{w}^{\prime} n
$$

suitable for describing the thin liquid flow along a guide plate as is shown on Fig. $1 \mathrm{~b}$. Here, $r, \phi, z$ are cylindrical coordinates, the functions $r_{w}=r_{w}(s), z_{w}=z_{w}(s)$ describe the geometry of the wall surface in terms of the length $s$ along the surface, $r_{w}^{\prime 2}+z_{w}^{\prime 2}=1$. The normal coordinate $n$ is 0 at the guide surface and $n=h$ at the free surface of the flow (here, $h=h(s)$ is the thickness of the metal layer). It is also convenient to introduce the normalized coordinate $\bar{n}$ (conformal to the flow surface) and the poloidal curvature $\kappa_{w}$ of the guide wall surface 


$$
\bar{n} \equiv \frac{n}{h}, \quad \kappa_{w} \equiv\left|r_{w}^{\prime \prime} z_{w}^{\prime}-z_{w}^{\prime \prime} r_{w}^{\prime}\right|
$$

In thin layer approximation we adopt the relations

$$
\kappa_{w} h \ll 1, \quad \frac{h}{R} \ll 1, \quad h \frac{\partial}{\partial s} \ll 1 .
$$

We also can neglect the normal component of velocity

$$
V \equiv \mathbf{V} \nabla s, \quad \mathbf{V} \nabla n \ll V
$$

The height $h$ of the stream is determined by the continuity equation

$$
\dot{M}=2 \pi \rho \int_{0}^{h} r V d n=2 \pi \rho h \int_{0}^{1} r V d \bar{n},
$$

where $\dot{M}$ is the mass flow in the stream.

The electric current can be represented through its stream functions as

$$
\mu_{0} \mathbf{j}=(\nabla F \times \nabla \phi), \quad j_{n}=\frac{F_{s}^{\prime}}{\mu_{0} r}, \quad j_{s}=-\frac{F_{\bar{n}}^{\prime}}{\mu_{0} h r}
$$

The stream function of the electric current $F$ includes three components

$$
F=F_{w}+F_{\text {ext }} \bar{n}+i(\bar{n}, s), \quad F_{w}=r B_{\text {tor }}=\text { const }
$$

The major term $F_{w}=0.2 I_{T F C}$ is related to the total current $I_{T F C}$ in the toroidal field coils, $F_{\text {ext }} \bar{n}$ represents the poloidal current (uniformly distributed across the stream), $F_{\text {ext }}=$ $0.2 I_{\text {pol }}$, driven through the stream by an external source and $i$ is the stream function of the eddy currents. Assuming wall thickness smaller than $h$ and neglecting the current sharing between the wall and the stream, $i$ satisfies the boundary conditions $i(0, s)=i(1, s)=0$.

In thin layer approximation the MHD equations can be written in terms of their projections as

$$
\begin{aligned}
\rho \frac{\partial V_{n}}{\partial t}+\rho \kappa_{w} V^{2} & =-\frac{p_{\bar{n}}^{\prime}}{h}-\rho g \frac{z_{\bar{n}}^{\prime}}{h}-\frac{F F_{\bar{n}}^{\prime}}{\mu_{0} h r^{2}} \\
\rho \frac{\partial V}{\partial t}+\rho V V_{s}^{\prime} & =-p_{s}^{\prime}-\rho g z_{w}^{\prime}-\frac{F F_{s}^{\prime}}{\mu_{0} r^{2}}+\frac{\nu}{h^{2}} V_{\bar{n} \bar{n}}^{\prime \prime} \\
-\frac{1}{h} \varphi_{E, \bar{n}}^{\prime}+\frac{V F}{r} & =0, \quad-\varphi_{E, s}^{\prime}=-\frac{F_{\bar{n}}^{\prime}}{\mu_{0} \sigma h r}
\end{aligned}
$$


All $s$-derivatives here are taken at $\bar{n}=$ const. Eqs. (11-13) together with Eq. (8) represent a full set of MHD equations. We kept the time derivative of the normal component of velocity in the first equation in order to cover also possible instabilities of the streams. Assuming that the flow is directed along the magnetic surfaces of the equilibrium configuration, we neglected here the interaction of the flow with the poloidal magnetic field. It can be easily included as an additive effects.

The normal component of Ohm's law in the leading approximation gives the distribution of the scalar potential

$$
\varphi_{E}=\varphi_{0}(s)-\frac{U F_{w}}{r^{2}}, \quad U \equiv-\int_{0}^{\bar{n}} h r V d \bar{n}
$$

while the longitudinal component

$$
\varphi_{0}^{\prime}-F_{w}\left(\frac{U}{r^{2}}\right)_{s}^{\prime}=\frac{F_{e x t}+i_{\bar{n}}^{\prime}}{\mu_{0} \sigma h r}
$$

determines the stream function $i(\bar{n}, s)$ and eddy currents

$$
\begin{aligned}
& \frac{i}{\mu_{0} \sigma h r}=-F_{w}\left(\frac{1}{r^{2}} \int_{0}^{\bar{n}} \tilde{U} d \bar{n}\right)_{s}^{\prime}, \quad \tilde{U} \equiv U-\int_{0}^{1} U d \bar{n} \\
& j^{n}=-\frac{\sigma F_{w}}{r}\left[h r\left(\frac{1}{r^{2}} \int_{0}^{\bar{n}} \tilde{U} d \bar{n}\right)_{s}^{\prime}\right]_{s}^{\prime}, \quad j^{s}=\sigma F_{w}\left(\frac{\tilde{U}}{r^{2}}\right)_{s}^{\prime} .
\end{aligned}
$$

The normal component of the equation of motion in leading order (neglecting the centrifugal force) gives the pressure distribution inside the fluid

$$
p=\frac{F^{2}(1)-F^{2}}{2 \mu_{0} r^{2}}=F_{w} \frac{F_{e x t}(1-\bar{n})-i}{\mu_{0} r^{2}} .
$$

Its substitution into the longitudinal equation of motion leads to a magnetic propulsion equation (MPE)

$$
\begin{aligned}
& \rho \frac{\partial V}{\partial t}+\rho V V_{s}^{\prime}=-\rho g z_{w}^{\prime}-\frac{I_{p o l} F_{w}^{2}(1-\bar{n})}{I_{T F C} \mu_{0}}\left(\frac{1}{r^{2}}\right)_{s}^{\prime} \\
& -\sigma h r F_{w}^{2}\left(\frac{1}{r^{2}} \int_{0}^{\bar{n}} \tilde{U} d \bar{n}\right)_{s}^{\prime}\left(\frac{1}{r^{2}}\right)_{s}^{\prime}+\frac{\nu}{h^{2}} V_{\bar{n} \bar{n}}^{\prime \prime} \\
& -\left(I_{\phi} B_{s}\right)_{s}^{\prime}(1-\bar{n})-\sigma V B_{n}^{2}
\end{aligned}
$$

which contains only one unknown variable $V(\bar{n}, s)$. 
The first term in the right hand side of MPE is gravity, the second is the magnetic propulsion term, which accelerates the fluid toward the low magnetic field side of the guide wall. The third term in the right hand side of MPE represents the diamagnetic drag force or "magnetic pumping", the fourth is viscosity. The last two terms, written without derivation describe interaction with the poloidal magnetic field. The first of them is the propulsion effect due to interaction of a toroidal current $I_{\phi}$ in the stream, if present, with tangential component of the poloidal field $B_{s}$, while the second is the drag force due to interaction with the normal (axisymmetric) $B_{n}$ component of the poloidal magnetic field (e.g., due to plasma displacement).

The diamagnetic drag force with respect to the propulsion term can be assessed assuming a uniform velocity profile across the stream. For values averaged over cross section, their ratio is determined by the propulsion parameter $R_{D}$ as

$$
\begin{aligned}
& \frac{\text { drag force }}{\text { propulsion }} \simeq \frac{R_{D} r_{w}^{\prime}}{3}, \quad R_{D} \equiv \Re_{2} \frac{I_{T F C}}{I_{p o l}}, \\
& \Re_{2} \equiv \frac{\mu_{0} \sigma h^{2} V}{r}
\end{aligned}
$$

The same propulsion parameter is responsible for possible peeling of the flow surface due to the appearance of "negative" pressure in the flow, if at some point $R_{D} r_{w}^{\prime}>1$.

For liquid lithium $\mu_{0} \sigma \simeq 4$ and for typical rector parameters, e.g., $r=6 \mathrm{~m}, V \simeq 20$ $\mathrm{m} / \mathrm{sec}, h \simeq 1 \mathrm{~cm}$, the diamagnetic drag force has only a small effect on flow. Even for $I_{\text {pol }}=0.01 I_{T F C}$, the propulsion parameter $R_{D} \ll 1$. It is important to notice that the Reynolds number $\Re_{2}$, introduced here, is different from the "electromagnetic" Reynolds number $\Re_{1} \equiv \mu_{0} \sigma h V$, which plays a role in plasma stabilization by the lithium streams $[6,7]$. This fact makes lithium dynamics consistent with the possible plasma stabilization by intense lithium streams.

The interaction with the normal component of the magnetic field is characterized by the conventional Reynolds number $\Re_{0}$

$$
\Delta \rho \frac{V^{2}}{2}=\Re_{0} \frac{1}{L} \int_{0}^{L} \frac{B^{2}}{2 \mu_{0}} d s, \quad \Re_{0} \equiv \mu_{0} \sigma L V,
$$


where $L$ is the length of the flow. Because $\Re_{0} \gg 1$, the flow is very sensitive to the quality of alignment of the flow with magnetic surfaces. Nevertheless, the magnetic propulsion allows a certain level of misalignment of the flow with the magnetic surfaces and of the normal magnetic field.

The possible electromagnetic instabilities appear in the next approximation when the centrifugal force and the dependence of the magnetic field line tension on the major radius are taken into account in the equation for pressure

$$
\left(p-\frac{F^{2}(1)-F^{2}}{2 \mu_{0} r^{2}}\right)_{\bar{n}}^{\prime}=-\rho g r_{w}^{\prime} h-\frac{2 p}{r} z_{w}^{\prime} h-\rho \kappa_{w} h V^{2}
$$

due to explicit dependence of the RHS on the height $h$ of the flow. Thus, the first term drives the gravitational instability (at the top of the vacuum chamber) [8], while the second one drives the "sausage" instability at the high field side, where $z_{w}^{\prime}<0$ [9]. The third term is the stabilizing effect of the centrifugal force.

The centrifugal force robustly stabilizes the sausage instability of the stream if

$$
\int_{-1}^{\bar{n}}\left(\rho \kappa_{w} V^{2}-2 p \frac{z_{w}^{\prime}}{r}\right) d \bar{n}>0, \quad \rho \frac{\left\langle V^{2}\right\rangle}{2}>\frac{p_{w}}{2 \kappa_{w} r} z_{w}^{\prime},
$$

where $p_{w}$ is the pressure of the stream on the wall surface. Because $\left|z_{w}^{\prime}\right|<1$ and the poloidal curvature is determined by the minor radius $a$ of the plasma, $k_{w} \simeq 1 / a$, this stability condition can be fulfilled for sufficiently high inlet stream velocity.

Much smaller flow speed is required for stabilization of the gravitational interchange instability

$$
\left\langle V^{2}\right\rangle>\frac{g\left|r_{w}^{\prime}\right|}{k_{w}}
$$

for the stream moving along the upper half of the vacuum chamber.

An example of a stationary solution to MPE (19) for liquid lithium is shown on Fig. 2. The velocity, averaged over the cross-section, and shown on Fig. 2a, rises from an inlet value to the characteristic level of $20 \mathrm{~m} / \mathrm{sec}$ at the middle of the path. The dotted line solution was obtained neglecting viscosity and the diamagnetic drag force in MPE. Neglecting diamagnetic drag force (dot-dashed line) shows the very small effect of diamagnetic drag (in 
contrast to the case of Ref. [10], where this effect was exaggerated). The normal component of magnetic field does affect the propulsion, as is shown by a dashed line solution. Note, that in this case the imposed vertical magnetic field would correspond $2-3 h(0)$ in the plasma displacement (depending on the plasma current).

The flow becomes significantly thinner on the way to the outlet point (Fig. 2b), Fig. 2c shows a comparison of the velocity profile for the cases without the effect of diamagnetic drag force, without both viscosity and drag force and with a normal magnetic field. Fig. 2d shows a comparison of the driven current density (solid lines) with the eddy currents (dot-dashed lines), which are significantly smaller at all cross-sections of the flow.

The effect of viscosity on magnetic propulsion in the laminar approximation is very weak and for typical velocities $10-20 \mathrm{~m} / \mathrm{sec}$ of the streams is localized in a sub-millimeter boundary layer near the surface of the wall. Note that, while magnetic propulsion drives the inner layer of the flow, viscosity propels the surface layer.

Summarizing, the magnetic propulsion of intense lithium streams represents an means for driving controlled lithium streams in a tokamak magnetic field. With development of the technology of injection/ejection of the streams it can be used for efficient power extraction from the plasma. The magnetic propulsion equation (19) describes the 2-dimensional MHD of the liquid lithium flow and shows robustness of the propulsion effect to viscous drag and diamagnetic drag force, characterized by dimensionless parameters $R_{D} \ll 1$ and $\Re_{2} \ll 1$. While the flow is sensitive to the normal components of the magnetic fields, in the case of magnetic propulsion misalignment by a few thicknesses of the flow is still tolerable. We also have shown that the streams can be stabilized by centrifugal force against "sausage" and gravitational instabilities. The thin layer approach can be extended to cases which would include disturbances of axisymmetry (like penetrations or non-symmetric normal fields).

This work was supported by United States Department of Energy Contracts No. DEAC02-76-CHO-3073. 


\section{REFERENCES}

[1] A. P. Babichev et al., Handbook of Physical Quantities (Edited by I. S. Grigoriev and E. Z. Melnikov, CRC Press, Boca Raton, New York, London, Tokyo, 1997), pp. $115,253,361,419,451$.

[2] V. A. Alexeev and I. T. Iakubov, Handbook of Thermodynamic and Transport Properties of Alkali Metals (Edited by R. W. Oshe, Blackwell Scientific Publications, Oxford, London, Edinburgh, Boston, Palo Alto, Melbourne, 1985), p. 730.

[3] M. A. Abdou et al., Fusion Engineering and Design 54, 181 (2001).

[4] R. Aymar, V. Chuyanov, M. Huguet, and Y. Shimomura, in Plasma Physics and Controlled Nuclear Fusion Research, Sorrento 2000 (IAEA, Vienna, 2000), p. OV/1.

[5] A. Jeffrey, Magnetohydrodynamics (Oliver \& Boyd, Edinburtgh and London, New York (Interscience Publishers Inc, A division of J. Willey \& sons, Inc., 1966), p. 90.

[6] L. E. Zakharov, Comments on Plasma Phys. and Controlled Fusion 2, 222 (2002).

[7] J. B. Taylor et al., Phys. of Plasmas 8, 4062 (2001).

[8] R. Dendy, Plasma Physics: an Introductory Course (Cambridge, University Press, 1993), p. 91.

[9] B. N. Trubnikov, Plasma Physics and the Problem of Controlled Thermonuclear Reactions (Ed. by M. A. Leontovich, Pergamon Press, New York, Oxford, London, Paris, 1961), Vol. 1, p. 349.

[10] A. Y. Aydemir, Phys. of Plasmas 8, 3411 (2001). 


\section{FIGURES}

FIG. 1. (a) Cross-section of a tokamak with the Toroidal Field Coils (TFC), a vacuum chamber with two lithium streams at the top and bottom halves of the vacuum chamber. (b) local coordinate system $n, s$.

FIG. 2. Numerical soluton of the MPE for $R=6 \mathrm{~m}, a=1.6 \mathrm{~m}, B_{\text {tor }}=5 \mathrm{~T}$ (at $r=R$ ), $I_{\text {ext }}=0.01 I_{T F C}=1.5 \mathrm{MA}, h_{\text {inlet }}=2 \mathrm{~cm}$. (a) averaged velocity and (b) height $h$ as function of longitudinal coordinate, (c) flow velocity profile in 3 cross sections along the stream. Solid lines represent solutions of MPE (19) for $B_{n}=0$, dashed lines for $B_{n}=0.005 B_{\text {tor }} \cos (s / a)$, dotted line corresponds the "ideal" solution with neither viscosity nor magnetic pumping, and dot-dashed lines the solution with no magnetic pumping. (d) profiles of externally driven current density (solid line) and of eddy current (dashed line). 


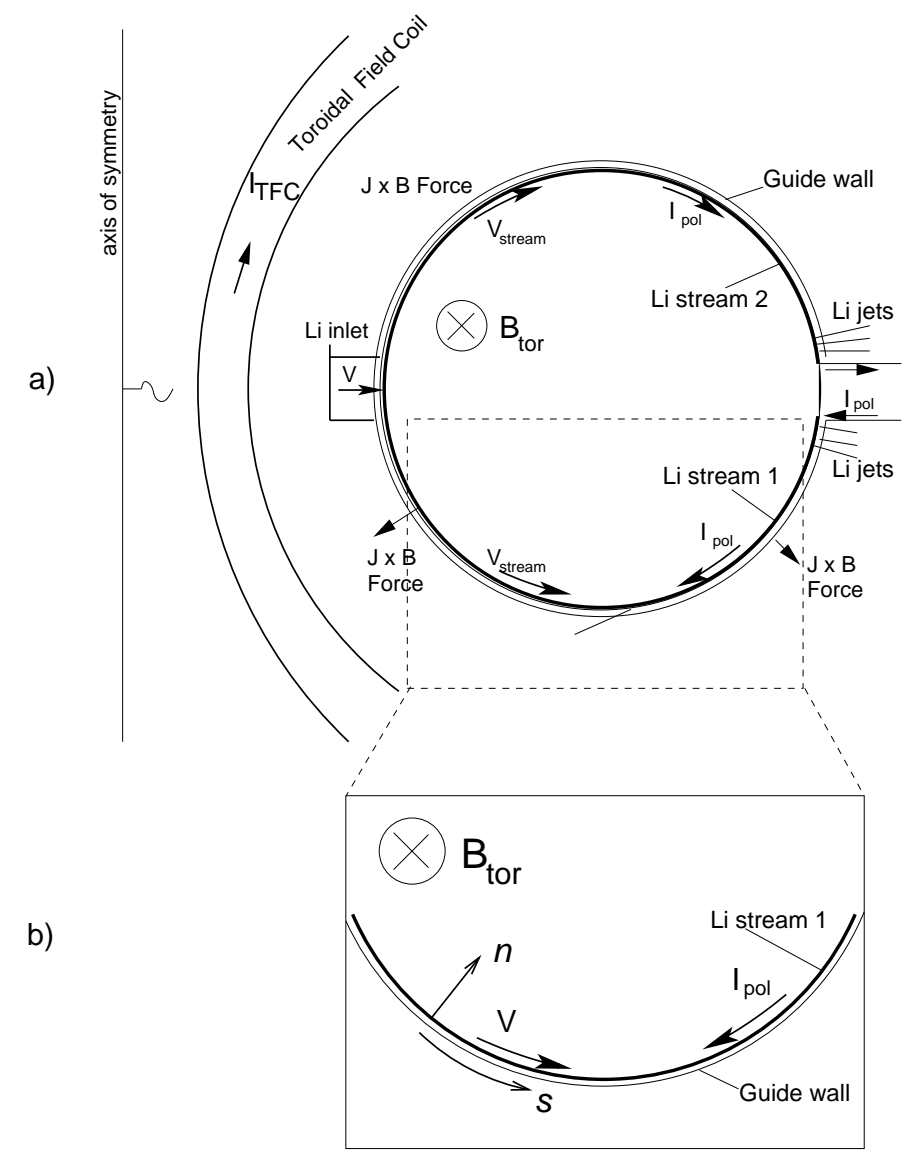


(a)

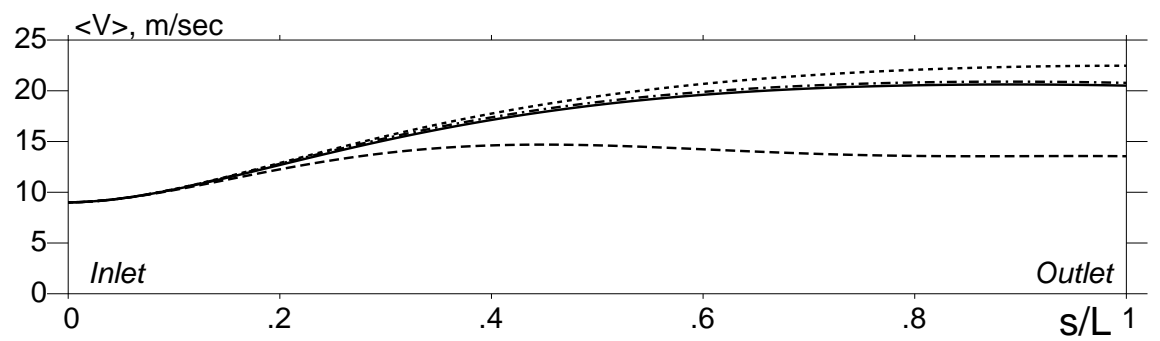

(b)

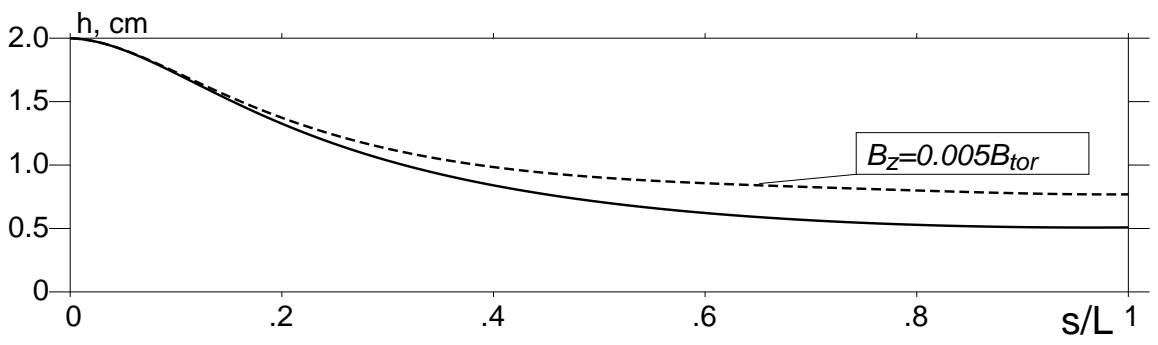

(c)
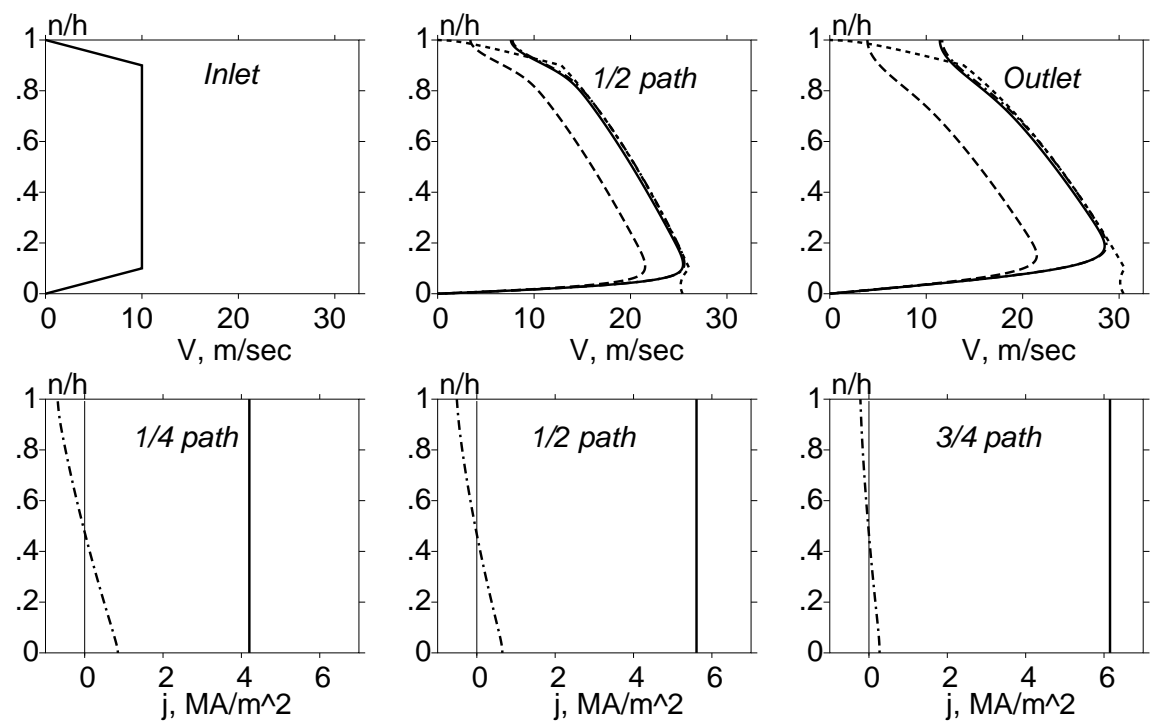


\section{External Distribution}

Plasma Research Laboratory, Australian National University, Australia

Professor I.R. J ones, Flinders University, Australia

Professor J oão Canalle, Instituto de Fisica DEQ/IF - UERJ , Brazil

Mr. Gerson O. Ludwig, Instituto Nacional de Pesquisas, Brazil

Dr. P.H. Sakanaka, Instituto Fisica, Brazil

The Librarian, Culham Laboratory, England

Library, R61, Rutherford Appleton Laboratory, England

Mrs. S.A. Hutchinson, JET Library, England

Professor M.N. Bussac, Ecole Polytechnique, France

Librarian, Max-Planck-Institut für Plasmaphysik, Germany

J olan Moldvai, Reports Library, MTA KFKI-ATKI, Hungary

Dr. P. Kaw, Institute for Plasma Research, India

Ms. P.J . Pathak, Librarian, Insitute for Plasma Research, India

Ms. Clelia De Palo, Associazione EURATOM-ENEA, I taly

Dr. G. Grosso, Instituto di Fisica del Plasma, Italy

Librarian, Naka Fusion Research Establishment, J AERI, J apan

Library, Plasma Physics Laboratory, Kyoto University, J apan

Research Information Center, National Institute for Fusion Science, J apan

Dr. O. Mitarai, Kyushu Tokai University, J apan

Library, Academia Sinica, Institute of Plasma Physics, People's Republic of China

Shih-Tung Tsai, Institute of Physics, Chinese Academy of Sciences, People's Republic of China

Dr. S. Mirnov, TRINITI, Troitsk, Russian Federation, Russia

Dr. V.S. Strelkov, Kurchatov Institute, Russian Federation, Russia

Professor Peter Lukac, Katedra Fyziky Plazmy MFF UK, Mlynska dolina F-2, Komenskeho Univerzita, SK-842 15 Bratislava, Slovakia

Dr. G.S. Lee, Korea Basic Science Institute, South Korea

Mr. Dennis Bruggink, Fusion Library, University of Wisconsin, USA

Institute for Plasma Research, University of Maryland, USA

Librarian, Fusion Energy Division, Oak Ridge National Laboratory, USA

Librarian, Institute of Fusion Studies, University of Texas, USA

Librarian, Magnetic Fusion Program, Lawrence Livermore National Laboratory, USA

Library, General Atomics, USA

Plasma Physics Group, Fusion Energy Research Program, University of California at San Diego, USA

Plasma Physics Library, Columbia University, USA

Alkesh Punjabi, Center for Fusion Research and Training, Hampton University, USA

Dr. W.M. Stacey, Fusion Research Center, Georgia Institute of Technology, USA

Dr. J ohn Willis, U.S. Department of Energy, Office of Fusion Energy Sciences, USA

Mr. Paul H. Wright, Indianapolis, Indiana, USA 
The Princeton Plasma Physics Laboratory is operated by Princeton University under contract with the U.S. Department of Energy.

\author{
Information Services \\ Princeton Plasma Physics Laboratory \\ P.O. Box 451 \\ Princeton, NJ 08543
}

Phone: 609-243-2750

Fax: 609-243-2751

e-mail: pppl_info@pppl.gov

Internet Address: http://www.pppl.gov 